\title{
Treatment of phalangeal osteomyelitis caused by a cat bite
}

\author{
Ji WooK Kim $^{1}$ | Joo Yong Kim ${ }^{2}$ ～Uk Gwan Kim ${ }^{1}$ | Dong Hee Kang ${ }^{1}$ | \\ Doo Sik Kim $^{1}$ | Ju Deok Kim ${ }^{1}$ @
}

\author{
${ }^{1}$ Department of Anesthesiology and \\ Pain Medicine, Kosin University \\ College of Medicine, Busan, Korea \\ ${ }^{2}$ Department of orthopaedics, Busan \\ Korea Hospital, Busan, Korea

\section{Correspondence} \\ Ju Deok Kim, Department of \\ Anesthesiology and Pain Medicine, \\ Kosin University College of Medicine, \\ 262 Gamcheon-ro, Seo-gu, Busan \\ 49267, Korea. \\ Email: uamyfriends@hanmail.net \\ Funding information \\ Not applicable
}

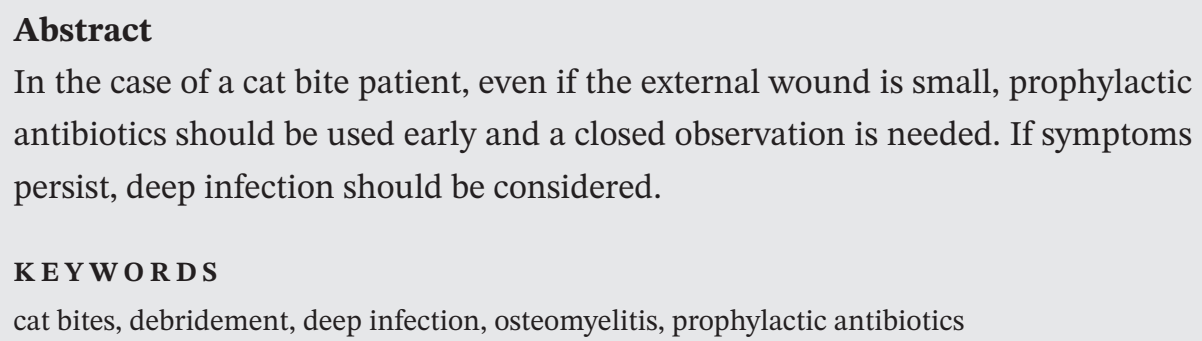

\section{1 | INTRODUCTION}

In the case of a cat bite patient, even if the external wound is small, prophylactic antibiotics should be used early and a closed observation is needed. Nevertheless, if symptoms persist and infection is present, aggressive treatment including debridement may be effective for a cat bite patient.

Animal bite injuries account for $1 \%$ of emergency department visits, of which $85 \%-90 \%$ are caused by dog bites. ${ }^{1}$ Cat bites are the second most common, accounting for $10 \%$ of bites by all animals. ${ }^{2}$ Although cat bites are relatively rare compared to dog bites, fatal complications such as wound infection and permanent damage can occur in about $30 \%-50 \%$ of all cat bite patients. There complications associated with cat bites are known to occur about twice as often as those associated with dog bites. ${ }^{2,3}$ The authors performed surgical treatment for osteomyelitis of the second middle phalanx in a patient with a cat bite. The treatment showed good results. Here, we report this case with a literature review.

\section{CASE REPORT}

A 44-year-old female patient visited the outpatient clinic with pain at the left middle phalanx of the second finger because she was bitten by a cat at home two days before the visit. The patient had a history of hepatitis C. She had no other special history or medical disease. On physical examination performed at the time of the first visit, mild swelling and redness were observed in the middle phalanx of the left second finger and approximately $1 \mathrm{~mm}$ of wounds presumed to be cat tooth marks were observed on the volar and dorsal side. The patient's blood tests were normal (C-reactive protein [CRP]: $0.1 \mathrm{mg} / \mathrm{dl}$, erythrocyte sedimentation rate [ESR]: $10 \mathrm{~mm} / \mathrm{hr}$, and white blood cell [WBC] count: $6.5 \times 10^{9} / \mathrm{L}$ ). As it was judged to be a very minor wound, no special suture except simple dressing was performed. Cefazedone $1 \mathrm{~g}$ was administered intravenously for 1 week, followed by oral use for 1 week. After one week of treatment, the wound recovered without any particular problem. However, edema, redness, and mild 
pain persisted. Although there was no serious complaint of pain, edema and redness continued until 2 months after the injury. Thus, blood tests and simple radiographic examination were performed again. WBC count, ESR, and CRP were still in the normal ranges. However, an osteolytic lesion was observed in the middle phalanx of the second finger on simple radiographic (X-ray) examination (Figure 1). This lesion had irregular borders with a local defect, especially in the anterior cortical bone. Subsequent MRI showed an abscess measuring $0.3 \times 0.3 \mathrm{~cm}$ in the distal middle phalanx with surrounding bone edema and soft tissue inflammation (Figure 2).

The authors diagnosed osteomyelitis and decided to operate. First, a zigzag incision was made in the volar side of the second finger. No particular infection was found in the subcutaneous layer. However, when the A4 pulley was incised and opened, the infected granulation tissue was easily identified along the flexor tendon. The synovial membrane of the flexor tendon had a defect presumably caused by the cat's tooth (Figure 3A). Under the flexor tendon, the anterior cortex of the metacarpal bone was penetrated and the medullary cavity was exposed (Figure 3B). Debridement of infected tissue surrounding the metacarpophalangeal joint, Gram-staining, and culture were done. The A4 pulley was then reconstructed, and primary sutures were performed (Figure 3C). After the surgery, the splint was applied until the wound was completely healed for 2 weeks. After the suture was removed, limited

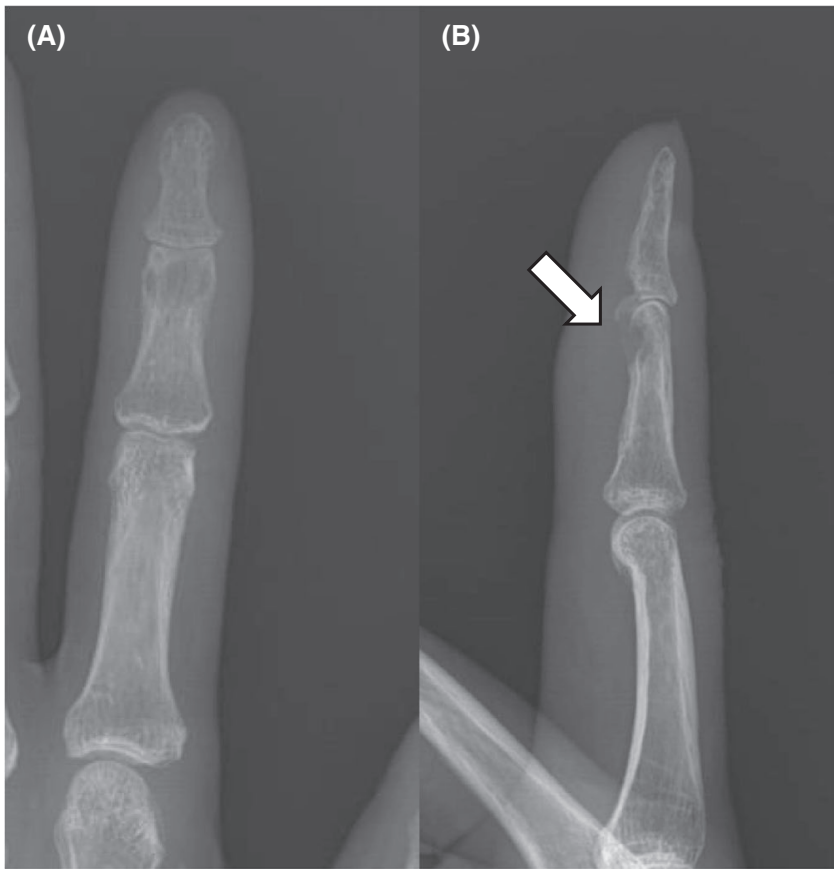

FIG URE 1 The loss of normal trabecular bone architecture and focal cortical loss (white arrow) on the volar side of the head of the 2nd middle phalanx was observed in the anterior-posterior (A) and lateral radiographs (B) taken at 2 months after injury

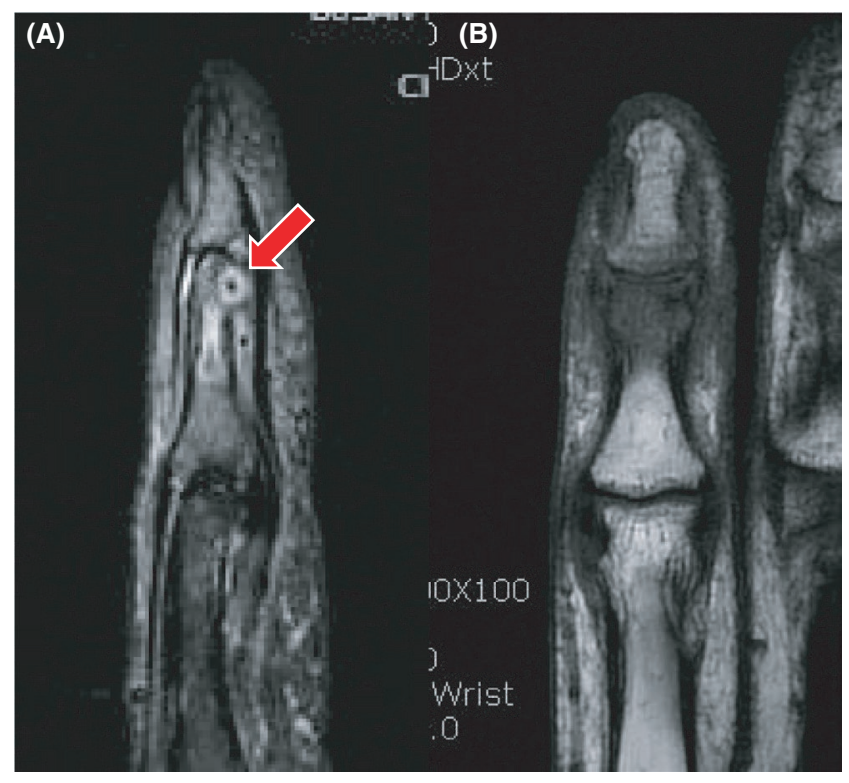

F I G U R E 2 In sagittal (A) and coronal view (B) of MRI, $0.3 \times 0.3 \mathrm{~cm}$ sized abscess (red arrow) with bone marrow edema and soft tissue inflammatory changes on the volar side of the head of the 2nd middle phalanx was observed

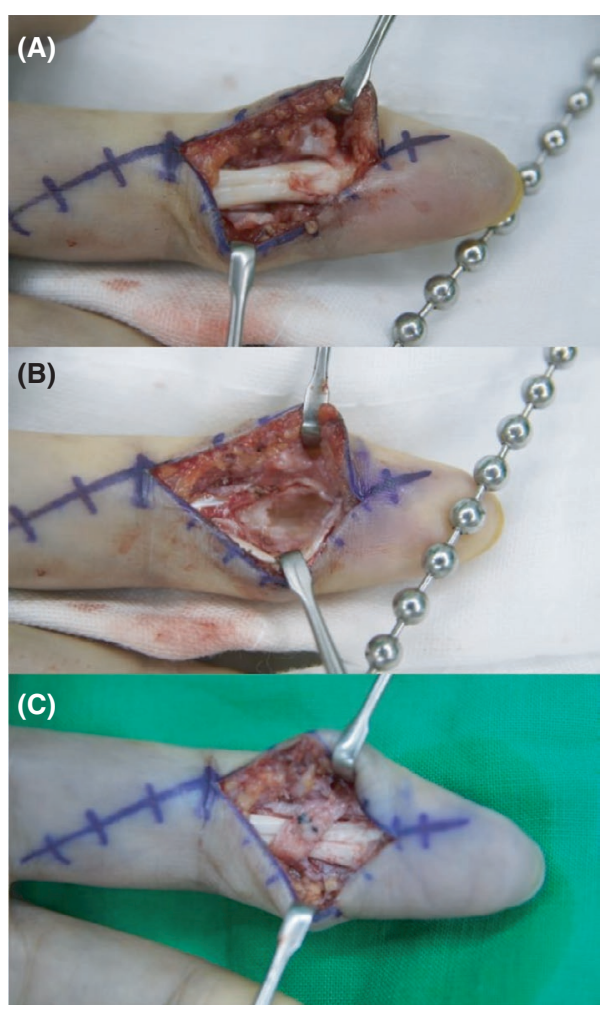

F I G U RE 3 (A) There were infected granulation tissues along the flexor tendon and partial tendon rupture caused by the teeth of a cat. (B) Under flexor tendon, there was a bony defect in volar side of the middle phalanx. Massive debridement of abscess and infected tissue was performed. (C) The wound was primarily repaired after A4 pulley repair 


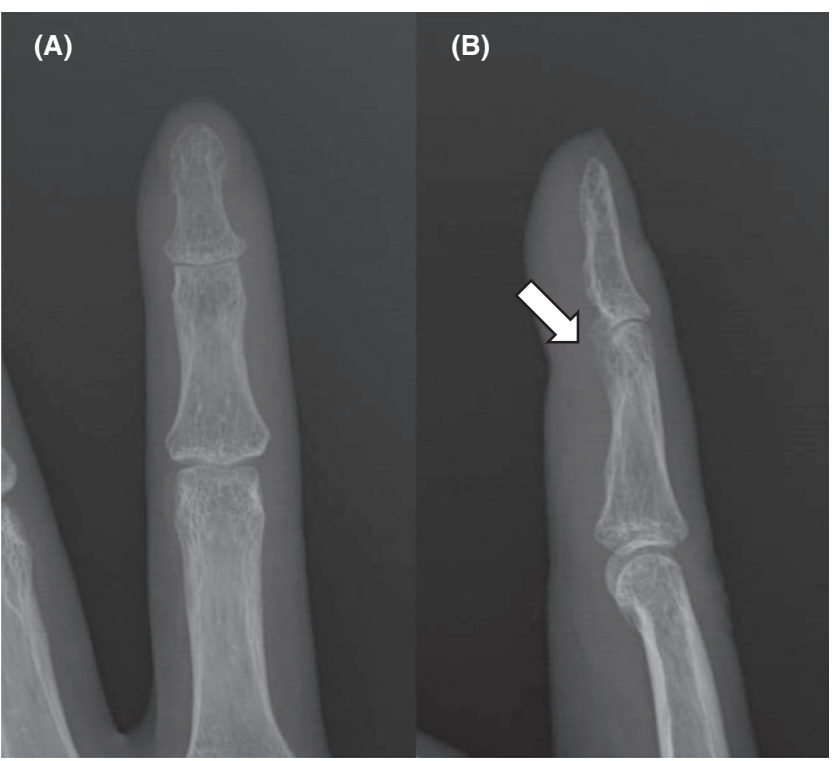

F I G U R E 4 Recovery of the bony defect (white arrow) was observed in the anterior-posterior (A) and lateral radiographs (B) of the 2nd middle phalanx taken at one year after operation. Range of motion of distal interphalangeal joint also restored to the normal range

joint movement was started. Range of motion exercise was started after all stitches were removed. For antibiotics, second-generation cephalosporin was administered intravenously up to 2 weeks after surgery. Oral antibiotics (cefaclor $250 \mathrm{mg}$ bid.) were then administered for four weeks thereafter. In the culture test performed for samples collected during surgery, no particular strain was identified even after culturing for more than four weeks. At 1year follow-up, radiographic examination showed that the previous bone defect had recovered (Figure 4) and joint motion was restored to the normal range. There were no signs of infection such as edema or fever.

\section{DISCUSSION}

Cat bites are the second most frequent animal bites. About $45 \%-63 \%$ of cat bites cause injuries to the hand. ${ }^{2}$ Compared with dog bites, cat bites have an incidence rate of less than $1 / 6$. However, the infection rate is more than doubled when a cat bite occurs. ${ }^{3}$

In animal bites, the occurrence of direct wounds by teeth and direct inoculation of bacteria occur more frequently than secondary infections by wounds. ${ }^{4}$ In the case of cat bites, the sharper and thinner teeth are the reason for this high rate of infection. Since cat tooth is easy to penetrate the joint capsule and bone, deep infections such as infectious arthritis and osteomyelitis can easily occur. Even if an infection occurs, it is often not detected early.
In case of a cat bite injury, strong cellulitis usually occurs locally within 12-24 h after the bite and an abscess may occur. Lim et al. ${ }^{5}$ have reported that local osteomyelitis may also occur. It has also been reported that $37 \%$ of cat bite patients require hospitalization and $12 \%$ of patients require surgical treatment. ${ }^{6}$

Pasteurella multocida is the most identified in results of culture for patients with cat bite wounds. ${ }^{7}$ This is because P. multocida is present in the normal flora of the oral cavity in more than $90 \%$ of felines. ${ }^{3}$ Westling et al. ${ }^{8}$ have performed a study on 78 cat bite patients and reported that P. multocida is identified in $70 \%$ of cases. Mitnovetski et al. ${ }^{6}$ have reported that $P$. multocida is found most frequently in culture of cat bite wounds. P. multocida can easily cause death. There are cases where it shows false negatives during culture. Serology itself cannot differentiate between current infection and past exposure. The fastest and most accurate identification method is polymerase chain reaction. However, even if there is a deep infection caused by a cat bite, $7 \%-8 \%$ of cases might not be culturable. ${ }^{9}$ In the present case, no bacteria were identified in the culture test performed for samples collected during surgery. However, false negatives cannot be ruled out. Talan et al. ${ }^{9}$ have reported that a bite wound can be considered to be infected if it meets one or more of three main criteria (fever, abscess, and lymphangitis) and if it meets 4 or more of five sub-criteria (erythema more than $3 \mathrm{~cm}$ from the margin of the wound, tenderness, edema, purulent discharge, and a white blood cell count of 12,000/ $\mathrm{ml}$ or more). Hospitalization should be considered for patients with severe cellulitis, systemic symptoms of infection (fever, vomiting, and confusion), clear evidence of bone, joint, ligament, or nerve involvement, rapid spread of infection within 24-48 h, or unresponsive to oral medications. ${ }^{2}$ However, there are cases with an incubation period for months or years without symptoms. In the case of our patient, there was no fever. In addition, ESR and CRP were normal. However, symptoms such as erythema, tenderness, and edema were found.

Mitnovetski et al. ${ }^{6}$ have reported that early and appropriate treatment is very important for patients with cat bites. If it is determined that an infection is present, immediate treatment is required. If osteomyelitis is suspected as in the present patient, surgical treatment and appropriate antibiotic treatment should be combined to remove the infected tissue. Treatment can be largely divided into treatment of wounds caused by direct damage and treatment of infection by the causative strain. It is based on opening the wound at an early stage. After that, whether to suture should be decided while observing the progress at intervals of 1-2 days. Early disinfection of the wound, keeping it open, and administering appropriate antibiotics usually yield good results. In order to use 
appropriate (accurate) antibiotics, a bacterial culture test is required. Since it takes $24-48 \mathrm{~h}$ to know the result of bacterial culture, prophylactic antibiotic treatment is necessary. Prophylactic antibiotic treatment after $48 \mathrm{~h}$ will result in poor prognosis. As a prophylactic antibiotic, penicillin is mainly used and it is more effective to prescribe the 2nd and 3rd generation cephalosporin class antibiotics than the 1st generation. ${ }^{6}$ In most patients, treatment is completed with only 10-14 days of antibiotic administration for superficial wounds. Intravenous administration of antibiotics for 6 weeks is required for wounds that have invaded bones or joints. The use of prophylactic antibiotics for small wounds can reduce the frequency of infection from $28 \%$ to $2 \% .{ }^{10}$ We administered the second-generation cephalosporin intravenously for up to 2 weeks after the surgery followed by oral antibiotics for 4 weeks.

For cat bite patients, it is effective to prescribe appropriate antibiotics before results of culture, even for wounds that are considered insignificant. It is also important to actively implement surgical incisions and debridement resections if infection is suspected.

\section{CONCLUSIONS}

In the case of a cat bite patient, even if the external wound is small, it is important to observe it closely while prescribing a prophylactic antibiotic. Nevertheless, if symptoms persist and infection is present, aggressive treatment including debridement may be effective for a cat bite patient.

\section{ACKNOWLEDGMENTS}

We would like to express our very great appreciation to professor Ryu for his valuable suggestions during the planning and development of this clinical case.

\section{CONFLICTS OF INTEREST}

"The authors declare no competing interests."

\section{AUTHOR CONTRIBUTIONS}

JW Kim, DS Kim, DH Kang, WK Kim, and JY KIM wrote the first draft of the manuscript. JD Kim corrected the final draft of the manuscript.

\section{ETHICAL APPROVAL}

Ethical approval was not mandatory for publication of case reports as per the institutional policy.

\section{PREVIOUS PRESENTATION IN CONFERENCES}

Not applicable.

\section{CONSENT}

The written informed consent was obtained from the patient for the publication of the text and images.

\section{DATA AVAILABILITY STATEMENT}

The data that support the findings of this study are available on request from the corresponding author. The data are not publicly available due to privacy or ethical restrictions.

\section{ORCID}

Ju Deok Kim (1) https://orcid.org/0000-0002-9236-5183

\section{REFERENCES}

1. Ellis R, Ellis C. Dog and cat bites. Am Fam Physician. 2014;90(4):239-243.

2. Hurt JB, Maday KR. Management and treatment of animal bites. JAAPA. 2018;31(4):27-31.

3. Freshwater A. Why your housecat's trite little bite could cause you quite a fright: a study of domestic felines on the occurrence and antibiotic susceptibility of Pasteurella multocida. Zoonoses Public Health. 2008;55(8-10):507-513.

4. Aziz H, Rhee P, Pandit V, Tang A, Gries L, Joseph B. The current concepts in management of animal (dog, cat, snake, scorpion) and human bite wounds. J Trauma Acute Care Surg. 2015;78(3):641-648.

5. Lim JS, Byun JH, Min KH, Lee HK, Choi YS. Osteomyelitis following domestic animal bites to the hand: two case reports and practical guidelines. Arch Plast Surg. 2016;43(6):590-594.

6. Mitnovetski S, Kimble F. Cat bites of the hand. Anz J Surg. 2004;74(10):859-862.

7. Lloret A, Egberink H, Addie D, et al. Pasteurella multocida infection in cats: $\mathrm{ABCD}$ guidelines on prevention and management. J Feline Med Surg. 2013;15(7):570-572.

8. Westling K, Farra A, Cars B, et al. Cat bite wound infections: a prospective clinical and microbiological study at three emergency wards in Stockholm, Sweden. J Infect. 2006;53(6):403-407.

9. Talan DA, Citron DM, Abrahamian FM, Moran GJ, Goldstein EJC. Bacteriologic analysis of infected dog and cat bites. N Engl J Med. 1999;340(2):85-92.

10. Medeiros I, Saconato H. Antibiotic prophylaxis for mammalian bites. Cochrane Database Syst Rev. 2001;(2):CD001738.

How to cite this article: Kim JW, Kim JY, Kim UG, Kang DH, Kim DS, Kim JD. Treatment of phalangeal osteomyelitis caused by a cat bite. Clin Case Rep. 2021;9:e04915. https://doi.org/10.1002/ ccr3.4915 